\title{
Solving characterization challenges for CdTe-based thin film photovoltaics: comparison of analytical techniques
}

\author{
K. Dovidenko, V.S. Smentkowski, O. Riccobono, L.A. Le Tarte, H. Piao, W. Heward, D. Ellis, R. \\ Neander, J.N. Johnson, B.A. Korevaar, J.A. DeLuca \\ GE Global Research Center, Niskayuna, NY 12309
}

In this work, we compare different characterization approaches to solving complex scientific challenges of CdTe-based thin film photovoltaics. We have employed a suite of characterization techniques including Transmission Electron Microscopy (TEM), Electron Energy Loss Spectrometry, Time-of-flight Secondary Ion Mass Spectroscopy (ToF-SIMS), Auger Electron Spectroscopy (AES), X-ray diffraction (XRD) X-ray photoelectron spectroscopy (XPS), and Focused Ion Beam techniques. The objective is to compare applicability of specific characterization techniques to specific aspects of CdTe-based solar analysis and show the complimentary nature of each of the techniques.

CdTe has received recently a lot of attention as a promising material for thin film solar cells. CdTe is a II-VI compound semiconductor with a direct optical bandgap of about $1.5 \mathrm{eV}$ which is almost perfectly matched to the solar spectrum for optimal photovoltaic energy conversion. A record $16.5 \%$, efficiency device was reported by NREL in 2001 [1].

Characterization challenges of CdTe-based solar cell stem partially from the nature of materials themselves and partially from the technological approaches for cell fabrication. The former is due to extreme susceptibility of CdTe to mechanical and radiation-induced damage. This susceptibility severely limits applicability of many electron- and ion-beam based characterization techniques, and creates significant challenges in sample preparation. The other side of the challenge, the technological processes, involves multiple steps causing numerous interconnected effects that are almost continuously changing materials structure. Multiple characterization efforts are underway in many research organizations, using some combination of the mentioned above characterization techniques. In this work, we will compare the results from broad range of techniques and summarize its applicability to various steps of the CdTe-based solar analysis.

[1] X. Wu, J.C. Keane, R.G. Dhere, C. DeHart, D.S. Albin, A. Duda, T.A. Gessert, S. Asher, D.H. Levi, and P. Sheldon, Proc. of 17th E-PVSEC (2001) 995 